\title{
AVALIAÇÃO DA UNIFORMIDADE DE DISTRIBUIÇÃO DE UM SISTEMA DE IRRIGAÇÃO POR GOTEJAMENTO EM INHAME (Dioscorea cayennensis Lam.)
}

\author{
Márcio Aurélio Lins dos Santos ${ }^{1 *}$, Daniella Pereira dos Santos ${ }^{1}$, Diogo Souza Silva ${ }^{1}$, Marcos dos Santos Silva ${ }^{1}$, \\ Pedro Henrique Silva Cavalcante ${ }^{1}$
}

${ }^{1}$ Universidade Federal de Alagoas, Campus Arapiraca. Av. Manoel Severino Barbosa, Bom Sucesso CEP: 57309-005. Arapiraca - AL

*Autor para correspondência: mal.santo@arapiraca.ufal.br

RESUMO: O objetivo do trabalho foi avaliar a uniformidade de distribuição de água em sistemas de irrigação por gotejamento em cultivo de inhame, nas condições edafoclimáticas do município de Taquarana (AL). 0 estudo foi realizado numa área rural da agricultura familiar cultivada com inhame. Foram avaliadas as uniformidades de distribuição de água nas subunidades de irrigação por gotejamento, foram utilizados para avaliação coletores em cada emissor para determinar a vazão, conforme metodologia de Keller e Karmeli (1975). Após coleta dos dados, estimaram-se os coeficientes de uniformidade de Christiansen (CUC); coeficiente de uniformidade de distribuição (CUD); coeficiente de uniformidade estatístico (CUE); coeficiente de uniformidade de Hart (CUH); eficiência de aplicação (Ea). Os coeficientes de uniformidade de distribuição (CUC, CUD, CUE, CUH) obtiveram classificação regular. Já à eficiência de aplicação (Ea) apresentou classificação ruim, com valores médios de $70 \%$. O coeficiente de uniformidade de Hart (CUH) foi semelhante ao coeficiente de uniformidade de Christiansen (CUC), indicando distribuição normal da lâmina de água aplicada.

Palavras-Chave: Irrigação; Coeficientes de Uniformidade; Eficiência.

\section{DISTRIBUTION UNIFORMITY EVALUATION OF THE DRIP IRRIGATION SYSTEM IN YAM (Dioscorea cayennensis Lam.)}

\begin{abstract}
The objective the work was to evaluate the uniformity of water distribution in drip irrigation systems in yam cultivation, on the conditions soil and climatic of the municipality of Taquarana (AL). The study was conducted in a rural area of cultivated family farms with yam. Were evaluated of the water distribution uniformity in the drip irrigation subunits, were used for evaluation collectors on each emitter to determine flow, according to the methodology Karmeli e Keller (1975). After data collection, were estimated the Christiansen uniformity coefficient (CUC); distribution uniformity coefficient (DUC); statistical uniformity coefficient (EUC); Hart uniformity coefficient (CUH); application efficiency (Ea). The distribution uniformity coefficient (CUC, DUC, EUC, HUC) received regular classification. Already on application efficiency (Ea) had bad rating, with average values of $70 \%$. The uniformity coefficient Hart (CUH) was similar to the coefficient of uniformity of Christiansen (CUC), indicating normal distribution of the water blade.
\end{abstract}

Keywords: Irrigation; Uniformity Coefficients; Efficiency.

\section{INTRODUÇÃO}

No Nordeste do Brasil a cultura do inhame (Dioscorea sp.), constitui alternativa agrícola potencial para ampliar o consumo no mercado interno e atender a demanda do mercado externo, bem como fonte de renda para os pequenos e médios agricultores familiares. A cultivar inhame da costa (Dioscorea cayennensis) é recomendada para o plantio comercial (Melo et al., 2012).

O inhame é uma cultura de importância econômica e social nas regiões tropicais, incluindo 0 nordeste do Brasil que produziu 38.256 toneladas em 2006 (IBGE, 2013), sendo os com maiores produções os Estados da Paraíba e de Pernambuco, o Estado de 
Alagoas ocupa a quarta posição. Dentre os principais fatores responsáveis por essa baixa produtividade, têm-se o manejo inadequado da cultura, a baixa fertilidade do solo, o manejo inadequado do solo e da água e as irregularidades climáticas (Santos, 1996). Vale ressaltar que dentre as tecnologias aplicadas para obter maiores produções, a irrigação é considerada a técnica de aplicação da água, no qual é necessária para manter o controle da umidade do solo, objetivando maiores rendimentos na agricultura irrigada.

Nos cultivos de sequeiro, quando bem conduzidos, pode varia de 12.000 a $15.000 \mathrm{~kg} \mathrm{ha}^{-1}$; nos cultivos irrigados, podem atingir de 20.000 a $25.000 \mathrm{~kg}$ ha $^{-1}$ (Santos et al., 2007). Apesar da importância dessa cultura para a região Nordeste, a sua produtividade é baixa, em torno de $11.000 \mathrm{~kg} \mathrm{ha}^{-1}$ decorrente de fatores como: indisponibilidade de material propagativo de boa qualidade, alto nível de infecção de doenças fúngicas, uso indiscriminado de agrotóxicos e de fertilizantes químicos, alta incidência e severidade de fitonematóides no solo e nas túberas sementes (Melo et al., 2012).

A cultura do inhame se desenvolve, satisfatoriamente, em clima tropical quente e úmido, sob condições de regime pluvial de 1.000 a 1.600 mm anuais, com temperatura ótima diária de 24 a $39^{\circ} \mathrm{C}$ e umidade relativa do ar de 60 a 70\% (Melo et al., 2012). Produzem bem em solos de textura arenosa e média, profundos, bem drenados e arejados, férteis e ricos em matéria orgânica, com pH de 5,5 a 6,0 (Santos et al., 2006).

Oplantiodoinhameemépocas demaiordemanda hídrica da cultura garante uma boa produtividade. Entretanto, os riscos climáticos, relacionados à mudança de temperatura e precipitação, balanço de água no solo, deixam os produtores vulneráveis à redução na produtividade. A irrigação complementar neste período ou para o manejo no período da entre safra, no qual o inhame alcança uma rentabilidade econômica maior, se constitui na prática agrícola que oferece maior segurança ao produtor (Sampaio et al., 2009).

As pesquisas relacionadas à irrigação do inhame no Brasil são poucas, sendo as maiorias desenvolvidas na região da Paraíba (Metri, 1997; Pereira, 1997), no Estado de Alagoas não existem estudos objetivos sobre a necessidade hídrica do inhame.

A uniformidade de distribuição de sistema de irrigação tem influência direta no manejo e na eficiência acarretando no desempenho da cultura no campo e no custo da irrigação. Uma baixa uniformidade de distribuição de água faz com que determinadas plantas irrigadas pelo sistema recebam mais água e adubo do que outras, resultando em um desenvolvimento desuniforme dentro da cultura. A avaliação da operação dos sistemas de irrigação está ligada a diversos parâmetros no desempenho, como vazão, tempo de irrigação e uniformidade de aplicação de água, nos quais são considerados fundamentais para tomadas de decisões em relação ao diagnóstico do sistema. Porém, aos produtores é considerada uma tarefa de pouca importância, mesmo quando disponibilizam de tecnologia, mas lhes faltam orientação e conhecimento (Silva e Silva, 2005).

Com a avaliação dos sistemas de irrigação tem-se o conhecimento da qualidade com que a irrigação está sendo realizada, a partir de uma gama de coeficientes de uniformidade de aplicação de água, os quais expressam a variabilidade de distribuição aplicada pelo sistema de irrigação. Os coeficientes de uniformidade o qual utilizam o desvio-padrão como medida de dispersão são os: coeficiente de uniformidade estatístico (CUE), coeficiente de uniformidade de Hart (CUH), eficiência padrão da HSPA (UDH) (Paulino et al., 2009). Quando a lâmina aplicada pelos emissores tem uma distribuição normal, o CUC será igual ao CUH e o CUD igual a UDH (Rocha et al., 1999).

0 primeiro coeficiente foi proposto por Christiansen (1942 apud Rezende et al., 2002), o qual adota o desvio médio absoluto como medida de dispersão (CUC). 0 mesmo autor em seu trabalho também cita o coeficiente de uniformidade de distribuição (CUD), proposto por Criddle et al. (1956 apud Rezende et al., 2002), o qual introduziu outra medida da uniformidade, considerando a razão entre a média do menor quartil e a lâmina média coletada, o baixo valor de CUD expressa uma perda de água por percolação profunda quando a lâmina mínima aplicada corresponde à lâmina necessária.

Objetivou-se com 0 trabalho avaliar a uniformidade de distribuição de água em sistemas de irrigação por gotejamento em cultivo de inhame, nas condições edafoclimáticas do município de Taquarana (AL).

\section{MATERIAL E MÉTODOS}

O trabalho foi desenvolvido numa área rural da agricultura familiar, localizado no município de Taquarana 
$(A L)$, entre as coordenadas geográficas $9^{\circ} 38^{\prime} 56^{\prime \prime}$ de latitude Sul e $36^{\circ} 29^{\prime} 49^{\prime \prime}$ de longitude Oeste e $246 \mathrm{~m}$ de altitude. $\mathrm{Na}$ área do experimento está implantada a cultura do inhame ( $D$. cayennensis), onde é constituída por uma subunidade de aproximadamente um hectare (1,0 ha), com comprimento de linhas secundárias de $58,0 \mathrm{~m}$ e as linhas de gotejadores de $90,0 \mathrm{~m}$. Os espaçamentos adotados foram de 1,00 m entre linhas de plantio e de 0,30 m entre gotejadores. Foram avaliadas as uniformidades de distribuição de água nas subunidades de irrigação por gotejamento, com a medição de vazão de todos os gotejadores de uma unidade operacional.

Para avaliação deste trabalho utilizou-se 0 sistema de irrigação localizada, que em cada emissor foram colocados coletores para determinar as vazões coletadas, medidas em provetas, com capacidade 0,20 litros, capazes de interceptar a emissão de cada gotejador. O tempo de coleta foi fixado em três minutos, utilizandose cronômetros com precisão de 0,01 segundos, e convertidas às vazões para litros por hora, cujo resultado é a média de quatro repetições. Com os dados de volume foi possivvel efetuar os cálculos das varáveis analisadas.

A recomendação para o trabalho foi através da metodologia de Keller e Karmeli (1975), considerando a média dos gotejadores que contribuíam com a planta (um gotejador por planta). As linhas laterais selecionadas, ao longo da linha de derivação, foram a primeira, as situadas a 1/3 e 2/3 do comprimento e a última linha lateral.

A uniformidade com que uma subunidade de irrigação localizada distribui a água foi avaliada mediante o Coeficiente de Uniformidade (CUD), no qual se baseou na metodologia apresentada por Merrian e Keller (1978), em que cada subunidade se elegeu a lateral mais distante e mais elevada de todas as tubulações terciárias, e as duas intermediárias, em cada lateral selecionou-se quatro emissores seguindo o mesmo critério, no total foram selecionados 16 pontos de coleta, constituídos por 16 emissores, um em cada ponto, no caso, foram analisados os emissores que contribuíam no fornecimento de água para a planta.

Com os dados coletados, estimaram-se os Coeficientes de Uniformidade de Christiansen (CUC); Coeficiente de Uniformidade de distribuição (CUD); Coeficiente de Uniformidade Estatístico (CUE); Coeficiente de Uniformidade de Hart (CUH); Eficiência de Aplicação (Ea).

Para a determinação do Coeficiente de Uniformidade de Christiansen do sistema (CUC) utilizou-se a metodologia de Christiansen (1942) através da Equação 1:

$\mathrm{CUC}=\left(1-\frac{\sum_{\mathrm{i}=1}^{\mathrm{n}}\left|\mathrm{Q}_{\mathrm{i}}-\mathrm{Q}\right|}{\mathrm{n} \mathrm{Q}}\right) \cdot 100$

Em que:

CUC = Coeficiente de uniformidade de Christiansen, em \%;

$Q_{i}=$ Vazão coletada em cada gotejador, em $\mathrm{L} \mathrm{h}^{-1}$;

$Q=$ Média das vazões coletadas de todos os gotejadores, em L $h^{-1}$;

$\mathrm{n}=$ Número de gotejadores analisados .

O Soil Conservation Service (1968) propôs a equação para cálculo do coeficiente de uniformidade de distribuição (CUD), que considera a média dos $25 \%$ menores valores de precipitação em relação à média total. Logo, o Coeficiente de Uniformidade de Distribuição (CUD) que é baseada na razão entre as vazões mínimas e médias dos emissores (Merrian e Keller, 1978) é determinada a partir da Equação 2:

$\mathrm{CUD}=\left(\frac{Q_{(\S \%}}{\mathrm{Q}_{\text {média }}}\right) \cdot 100$

Em que:

CUD = Coeficiente de Uniformidade de Distribuição, em \%;

$Q_{(25 \%)}=$ Média de $25 \%$ do total de gotejadores com as menores vazões, em $\mathrm{L} \mathrm{h}^{-1}$;

$Q_{\text {média }}=$ Média das vazões coletadas nos gotejadores na subárea, em $\mathrm{L} \mathrm{h}^{-1}$.

A interpretação dos valores do CUD baseou-se na metodologia proposta por Merrian e Keller (1978): Excelente: maior que $90 \%$; bom: entre $80 \%$ e $90 \%$; regular: $70 \%$ e $80 \%$; e, ruim: menor que $70 \%$.

A uniformidade de aplicação de água para Coeficiente de Uniformidade Estatístico (CUE), foi determinado pela Equação 3 e a interpretação dos valores do coeficiente de uniformidade Estatístico baseou-se na metodologia apresentada por Bernardo e Mantovani (2009) que está apresentada na Tabela 1. 
Através da Equação 3 pode-se determinar 0 Coeficiente de Uniformidade Estatístico (CUE):

$\mathrm{CUE}=\left(1-\frac{\mathbb{d}}{\mathrm{Q}_{\text {média }}}\right) .100$

Em que:

CUE = Coeficiente de Uniformidade Estatístico, em \%;
$\mathrm{Sd}=$ Desvio-padrão dos valores de precipitação, em L h${ }^{-1}$; $Q_{\text {média }}=$ Média das vazões coletadas nos gotejadores na subárea, em $\mathrm{L} \mathrm{h}^{-1}$.

A classificação dos indicadores de desempenho do sistema de irrigação foi feita seguindo critérios e limites estabelecidos pela literatura específica (Tabela 1).

Tabela 1. Interpretação dos valores do CUC, CUD e CUE

\begin{tabular}{cccc}
\hline CLASSIFICAÇÃO & CUC (\%) & CUD (\%) & CUE (\%) \\
\hline Excelente & $>90$ & $>84$ & $90-100$ \\
Bom & $80-90$ & $68-84$ & $80-90$ \\
Razoável & $70-80$ & $52-68$ & $70-80$ \\
Ruim & $60-70$ & $36-52$ & $60-70$ \\
Inaceitável & $<60$ & $<36$ & $<60$ \\
\hline
\end{tabular}

Fonte: Bernardo e Mantovani (2009)

O Coeficiente de Uniformidade de Hart (Hart, 1961) utiliza um coeficiente de uniformidade usando, também, o desvio-padrão como medida de dispersão que é calculado através da Equação 4

$\mathrm{CUH}=\left(1-\sqrt{\frac{2}{\pi}} \cdot \frac{d}{\mathrm{Q}_{\text {média }}}\right) .100$

Em que:

$\mathrm{CUH}=$ Coeficiente de Uniformidade de Hart, em \%;

$\mathrm{Sd}=$ Desvio-padrão dos valores de precipitação, em $\mathrm{L} \mathrm{h}^{-1}$; $Q_{\text {média }}=$ Média das vazões coletadas nos gotejadores na subárea, em $L h^{-1}$.

A determinação da eficiência de irrigação para o método de irrigação localizada é de extrema dificuldade, mesmo o intervalo de irrigação sendo de vários dias. Entretanto, fazendo um manejo adequado das irrigações para reabastecer toda a água consumida pelas plantas e pelas perdas por percolação profunda irão variar em aproximadamente 10\%. Logo, a Eficiência de Aplicação (EA) da irrigação plena segundo Merrian e Keller (1978), pode ser estimada pela Equação 5:

$$
\mathrm{E}=\left(\frac{Q_{\text {média }}}{\mathrm{Y}_{\mathrm{a}}}\right) \cdot 100
$$

\section{Em que:}

Ea = Eficiência de Aplicação, em \%

$Q_{\text {média }}=$ Média das vazões coletadas nos gotejadores na subárea, em $\mathrm{L} \mathrm{h}^{-1}$;

Ya = lâmina média aplicada pelos gotejadores, em $\mathrm{mm}$

\section{RESULTADOS E DISCUSSÃO}

Após a coleta das vazões, pode-se observar na Tabela 2 que a maioria dos gotejadores apresentou vazões abaixo do recomendado pelo o fabricante que é de $1,56 \mathrm{~L} \mathrm{~h}^{-1}$, o que permite afirmar que a irrigação nesta área está abaixo do esperado.

Tabela 2. Valores da determinação de vazão (q) das linhas gotejadoras, para determinação dos Coeficientes de Uniformidade para a cultura do inhame

\begin{tabular}{|c|c|c|c|c|}
\hline \multirow{3}{*}{ EMISSOR POR LINHA } & \multicolumn{4}{|c|}{ LINHA LATERAL DE DERIVAÇÃO } \\
\hline & $1^{\text {a }}$ Linha & $20^{\mathrm{a}}$ Linha & $40^{\mathrm{a}}$ Linha & $60^{\mathrm{a}}$ Linha \\
\hline & $q\left(L h^{-1}\right)$ & $q\left(L h^{-1}\right)$ & $q\left(L h^{-1}\right)$ & $q\left(L h^{-1}\right)$ \\
\hline $1^{\circ}$ Gotejador & 1,28 & 0,86 & 1,31 & 0,75 \\
\hline $67^{\circ}$ Gotejador & 0,70 & 0,80 & 1,43 & 0,75 \\
\hline $134^{\circ}$ Gotejador & 0,45 & 0,76 & 1,44 & 0,97 \\
\hline $200^{\circ}$ Gotejador & 0,64 & 1,12 & 1,33 & 1,25 \\
\hline VAZÃO MÉDIA & 0,76 & 0,88 & 1,38 & 0,93 \\
\hline
\end{tabular}


A análise conjunta dos coeficientes de uniformidade é essencial para avaliar o desempenho de quaisquer sistemas de irrigação (Santos et al., 2013; Rodrigues et al., 2013). Na Tabela 3 encontra-se o Coeficiente de Uniformidade de Christiansen (CUC) encontrado para a cultura do inhame foi considerado regular pela classificação da (Asae, 1996). O valor mínimo do Coeficiente de Christiansen aceitável em sistema de irrigação por gotejamento é de $80 \%$ (Bernardo e Mantovani, 2009). Os Coeficientes de Uniformidade de Distribuição (CUD) e Estatístico (CUE) obtiveram classificação regular (Tabela 3).

Tabela 3. Valores e classificação dos coeficientes de uniformidade de distribuição de água em campo do sistema de irrigação por gotejamento (Asae, 1996)

\begin{tabular}{c|c|c}
\hline MÉTODO & EFICIÊNCIA & CLASSIFICAÇÃO \\
\hline $\operatorname{CUC}(\%)$ & 70,85 & Regular \\
\hline $\operatorname{CUD}(\%)$ & 63,82 & Regular \\
\hline $\operatorname{CUE}(\%)$ & 65,69 & Regular \\
\hline $\operatorname{CUH}(\%)$ & 71,88 & Regular \\
\hline $\operatorname{EA}(\%)$ & 54,98 & Ruim \\
\hline
\end{tabular}

0 valor do coeficiente de uniformidade de Christiansen (CUC), segundo Bernardo et al. (2009), está classificado como excelente pois são superiores a $90 \%$ e recomenda um valor mínimo admitido de $80 \%$ dentro da parcela de irrigação. $O$ valor do coeficiente de uniformidade de distribuição (CUD) do sistema de irrigação localizada por gotejamento gravitacional avaliado em campo 96,61\% encontra-se classificado como excelente segundo classificação proposta por Keller e Karmeli (1974) e Bralts (1986) a qual especifica como tal valores de CUD superiores a 90\% (Valnir Júnior et al., 2012). O CUD é o mais utilizado na avaliação, pois este possibilita uma medida mais restrita, dando maior peso às plantas que recebem menos água, sendo o mesmo adotado para o cálculo do tempo de irrigação (López et al., 1992); Frizzone e Dourado Neto, 2003).

A avaliação do valor de CUE de 95,78\% foi classificada como excelente de acordo com a classificação proposta por Favetta e Brotel (2001) onde determina os valores de CUE como: excelente maior igual a $90 \%$, muito bom entre 80 e $90 \%$, regular entre 70 e $79 \%$, péssimo entre 60 e $69 \%$ e inaceitável menor que 60\% (Valnir Júnior et al., 2012).

Estudos com duas metodologias de amostragem: Keller e Karmelli (1975) com 16 pontos e Deniculi (1980) com 32 pontos, obtiveram valores do CUC e do CUD superiores a 80\%, com isso o sistema foi classificado como bom. Utilizando o teste $F$, comparando o CUC de cada metodologia de amostragem, mostrou que estatisticamente são diferentes, e comparando o CUD, mostrou que são iguais, mas ambos com a mesma classificação, os valores de coeficiente de variação de 0,01 para duas metodologias e de desvio padrão de 0,00645 para Keller e Karmelli e 0,00621 para Deniculi (Szekut et al., 2012).

Ainda se pode observar que o Coeficiente de Uniformidade de Hart (CUH) pareou-se ao do Coeficiente de Uniformidade de Christiansen (CUC). $\mathrm{Na}$ irrigação por aspersão quando a lâmina de água aplicada tem distribuição normal, tem-se que o CUH é igual ao CUC (Hart, 1961). Neste trabalho pode-se observar o mesmo fato para irrigação por gotejamento. Vale ressaltar que o coeficiente de uniformidade de Christiansen (CUC) é um índice que faz uso do desvio médio absoluto, para expressar a dispersão das lâminas (Rezende et al., 2002).

O sistema apresentou uma eficiência de aplicação (EA) de 54,98\%, muito abaixo ao valor recomendado (Tabela 3), que deve estar entre 90 a 95\% (Bernardo e Mantovani, 2009). Em muitas situações as causas da ineficiência de um sistema de irrigação podem ser várias, dentre elas: o entupimento de gotejadores, pouca pressão de serviço nas linhas de derivação, sistemas de irrigação velhos, entre outros. Avaliando sistema de irrigação por gotejamento na cultura do cafeeiro, semelhantemente, obteve baixa eficiência de aplicação, sendo explicada pela ausência do manejo da irrigação na área estudada. A lâmina aplicada no período avaliado é superior à lâmina real necessária à cultura irrigada, aplicando-se água em excesso (Rodrigues et al., 2013). 
Apesar de bons índices de uniformidade $e$ eficiência se faz necessário à observação do sistema, às vezes a eficiência de aplicação de água se encontrada abaixo do recomendado (Santos et. al., 2013). A melhoria do desempenho e da eficiência de aplicação de um sistema de irrigação pode ser obtida por meio da adoção de práticas de manejo, como limpeza periódica do sistema de filtragem, dos emissores e das linhas laterais (Rodrigues et al., 2013).

A aplicação dos valores de uniformidade como referência do que está ocorrendo com os sistemas de irrigação localizada ao longo de seu uso, as diferenças porcentuais encontradas mostram-se muito baixas, confirmando a validade das equações propostas, e viabilizando a comparação entre resultados de uniformidade dos diferentes métodos utilizados, através da estimativa desses coeficientes de uniformidade, sendo 0 uso dessas equações potencialmente interessante na avaliação pós implantação de sistemas de irrigação (Favetta e Brotel, 2001).

Logo, os resultados obtidos do sistema de gotejamento avaliado apresentaram um desempenho regular para os coeficientes de uniformidade e ruim para eficiência de aplicação de um sistema por gotejamento. 0 coeficiente de uniformidade de Hart (CUH) pareouse ao do Coeficiente de Uniformidade de Christiansen (CUC), indicando uma distribuição normal da lâmina de água aplicada.

\section{REFERÊNCIAS BIBLIOGRÁFICAS}

Asae (American Society of Agricultural Engineers). Standard engineering practices data: EP458. Field evaluation of microirrigation systems. St. Joseph: ASAE 1996, 972-797.

BRALTS, V.F. Field performance and evaluation. In: Nakayama, F.S.; Bucks, D.A. (Ed.) Trickle irrigation for crop production. Development in Agricultural Engineering, 9. Amsterdam: Elsevier, 1986, 216-240.

BERNARDO, S.; SOARES, A. A.; MANTOVANI, C. Manual de irrigação, edição: $8^{a}$. Editora: UFV, $2^{a}$ reimpressão. Viçosa, MG, 2009, 625p.

CHRISTIANSEN, J. E. Irrigation by sprinkling. Berkely: Universit of California, 1942.124p.
CRIDDLE, W.D.; DAVIS, S.; PAIR, C.H.; SHOCKLEY, D.G. Methods for evaluating irrigation systems. Soil Conservation Service (USDA), Agricultural Handbook, Washington DC. 82, 1956, 24p.

DENÍCULI, W.; BERNARDO, S.; THIÉBAUT, J.T.L.; SEDIYAMA, G.C. Uniformidade de distribuição de água, em condições de campo num sistema de irrigação por gotejamento. Revista Ceres. 1980, 27, 150, 155-162.

FAVETTA, G. M.; BOTREL, T. A. Uniformidade de sistemas de irrigação localizada: validação de equações. Scientific Agricola. 2001, 58, 427-430.

FRIZZONE, J. A.; DOURADO NETO, D. Avaliação de sistemas de irrigação. In: Miranda, J. H. de; Pires, R. C. de M. (Org.). Irrigação. Série Engenharia Agrícola, 2: FUNEP. Piracicaba, SP, 2003. 573-651.

HART, W. E. Overhead irrigation pattern parameters. Transactions of the ASAE, Saint Joseph, 1961, 42, 7, 354-355.

IBGE. Sistema IBGE de recuperação automática SIDRA. (http://sidra.ibge.gov.br). (acesso: 20 novembro 2013).

KELLER, J.; KARMELI, D. Trickle irrigation design parameters.Transactions of the ASAE, St. Joseph, $1974,17,4,678-684$

KELLER, J.; KARMELI, D. Trickle irigation desing. Rain Bird Sprinnkler Manufacturing Corporation, 1975, 133p.

LÓPEZ, J. R., ABREU, J. M. H.; REGALADO, A.P.; HERNÁNDEZ, J. F. G. Riego Localizado. Madrid, Espana: Mundi - Prensa, 1992. 405p

MELO, R. F.; DE, ANJOS, J. B. DOS; COELHO, L. C.; SILVA, F. P. DA. Impacto da adubação orgânica no rendimento do inhame da costa (Dioscorea cayennensis) em sistema irrigado. Fertibio 2012: A responsabilidade socioambiental da pesquisa agrícola. Maceió, AL, 2012.

MERRIAN, J. L.; KELLER, J. Farm irrigation system evaluation: a guide for management. Logan; Utah State University, 1978, 271p. 
METRI, J. E. C. Necessidades hídricas e produtividade da cultura do inhame (Cará-da-Costa) irrigado no Estado da Paraíba. Tese de doutorado do CCT/UFPB. Campina Grande, PB. 1997. 113 p.

PAULINO, M. A. DE O.; FIGUEIREDO, F. P. DE; FERNANDES, R. C.; MAIA, J. T. L. S.; GUILERME, D. DE O.; BARBOSA, F. S. Avaliação da uniformidade e eficiência de aplicação de água em sistemas de irrigação por aspersão convencional. Revista Brasileira de Agricultura Irrigada. 2009, 3, 2, 48-54.

PEREIRA, J. R. B. Estudo da umidade do solo na fase de Germinação do Cará-da-Costa (Dioscorea cayennensis Lam.). Coeficientes Hídricos. Dissertação (Mestrado do CPGEAG/CCT/UFPB). Campina Grande, $\mathrm{PB}, 1997.87 \mathrm{p}$.

REZENDE, R.; GONÇALVES, A. C. A.; FREITAS, P. S. L.; FRIZZONE, J. A.; TORMENA, C. A.; BERTONHA, A. Influência da aplicação de água na uniformidade da umidade no perfil do solo. Acta Scientiarum. 2002, 24, 5, 1553-1559.

ROCHA, E. M. M; COSTA, R. N. T.; MAPURUNGA, S. M. S. CASTRO, P. T. Uniformidade de distribuição de água por aspersão convencional na superfície e no perfil do solo. Revista Brasileira de Engenharia Agrícola e Ambiental (AGRIAMBI). 1999, 3, 2, 154-160.

RODRIGUES, R. R.; COLA, M. P. A.; NAZÁRIO, A. A.; AZEVEDO, J. M. G. DE; REIS, E. F. DOS. Eficiência e uniformidade de um sistema de irrigação por gotejamento na cultura do cafeeiro. Ambiência Guarapuava (PR). 2013, 9, 2, 323-334.

SAMPAIO, A. H. R.; COELHO FILHO, M. A.; COELHO, E. F.; MACHADO, E. S.; SILVA, T. S. M. DA; SANTOS, M. B. Evapotranspiração da cultura do inhame (Dioscorea cayennensis) nas condições de Cruz das Almas - BA. $\mathrm{XVI}$ Congresso Brasileiro de Agrometeorologia. Belo Horizonte, MG. 2009.
SANTOS, C. S. DOS.; SANTOS, D. P. DOS; SILVA, P. F. DA.; ALVES, E. DA S.; SANTOS, M. A. L. Avaliação da uniformidade de distribuição de um sistema de irrigação por gotejamento. Revista Verde. 2013, 8, 3, 10-16.

SANTOS, E. S. DOS. Inhame (Dioscorea spp.): aspectos básicos da cultura. Emepa-Sebrae. João Pessoa, PB. 1996, 158 p.

SANTOS, E. S. DOS; CAZÉ FILHO, J.; LACERDA, J. T. DE; CARVALHO, R. A. Inhame (Dioscorea sp.) Tecnologias de Produção e Preservação Ambiental. Tecnologia \& Ciências Agropecuárias. 2007, 1, 1, 31 36.

SANTOS, E. S. DOS; CAZÉ FILHO, J.; LACERDA, J. T. DE; CARVALHO, R. A.; FONTINÉLLI, I. S. C.; SILVA, J. B. DA; BARBOSA, M. M.; CASSIMIRO, C. M. Inhame e preservação ambiental. Embrapa-Emepa João Pessoa, PB. 2006. 6 p.

SILVA, C. A. DA; SILVA, C. J. Avaliação de uniformidade em sistemas de irrigação localizada. Revista Científica Eletrônica de Agronomia. 2005, n. 8. 17p.

Soil Conservation Service. National Engineering Handbook. Washington: Sprinkler Irrigation, Section 15, Chapter 11. 1968, 83p.

SZEKUT, F. D.; KLEIN, M. R.; REIS, C. F.; SUSZEK, F. L.; VILAS BOAS, M. A. Uniformidade de um sistema de irrigação por gotejamento destinado à agricultura familiar. I Inovagri, Fortaleza, CE. 2012.

VALNIR JÚNIOR, M.; SOUSA, L. S. DE.; CARVALHO, C. M. DE; RAMOS, M. J. B.; LUNA, N. DE S.; ARAÚJO, O. P. Desempenho de um sistema de irrigação por gotejamento gravitacional em campo com a cultura da abóbora. I Inovagri, Fortaleza, CE. 2012. 